\title{
Automating Venous Thromboembolism Risk Calculation Using Electronic Health Record Data Upon Hospital Admission: The Automated Padua Prediction Score
}

\author{
Pierre Elias, $\mathrm{MD}^{1,2^{*}}$, Raman Khanna, MD², Adams Dudley, MD, MBA ${ }^{3}$, Jason Davies, MD, PhD4, Ronald Jacolbia, MSN², \\ Kara McArthur, BA ${ }^{5}$, Andrew D. Auerbach, $\mathrm{MD}^{2}$
}

\begin{abstract}
${ }^{1}$ Department of Medicine, Columbia University Medical Center, New York, New York; ${ }^{2}$ Division of Hospital Medicine, University of California San Francisco, San Francisco, California; ${ }^{3}$ Philip R. Lee Institute for Health Policy Studies, University of California, San Francisco, San Francisco, California; ${ }^{4}$ Department of Neurological Surgery, University of California, San Francisco, San Francisco, California; ${ }^{5}$ Abramson Center for the Future of Health, University of Houston, Houston, Texas.
\end{abstract}

\begin{abstract}
BACKGROUND: Venous thromboembolism (VTE) risk scores assist providers in determining the relative benefit of prophylaxis for individual patients. While automated risk calculation using simpler electronic health record (EHR) data is feasible, it lacks clinical nuance and may be less predictive. Automated calculation of the Padua Prediction Score (PPS), requiring more complex input such as recent medical events and clinical status, may save providers time and increase risk score use.
\end{abstract}

OBJECTIVE: We developed the Automated Padua Prediction Score (APPS) to auto-calculate a VTE risk score using EHR data drawn from prior encounters and the first 4 hours of admission. We compared APPS to standard practice of clinicians manually calculating the PPS to assess VTE risk.

DESIGN: Cohort study of 30,726 hospitalized patients. APPS was compared to manual calculation of PPS by chart review from 300 randomly selected patients.
MEASUREMENTS: Prediction of hospital-acquired VTE not present on admission.

RESULTS: Compared to manual PPS calculation, no significant difference in average score was found (5.5 vs. 5.1, $P=0.073$ ), and area under curve (AUC) was similar (0.79 vs. 0.76 ). Hospital-acquired VTE occurred in $260(0.8 \%)$ of 30,726 patients. Those without VTE averaged APPS of 4.9 (standard deviation [SD], 2.6) and those with VTE averaged 7.7 (SD, 2.6). APPS had AUC $=0.81$ (confidence interval $[\mathrm{Cl}], 0.79-0.83$ ) in patients receiving no pharmacologic prophylaxis and $\mathrm{AUC}=0.78(\mathrm{Cl}, 0.76$ 0.82 ) in patients receiving pharmacologic prophylaxis.

CONCLUSION: Automated calculation of VTE risk had similar ability to predict hospital-acquired VTE as manual calculation despite differences in how often specific scoring criteria were considered present by the 2 methods. Journal of Hospital Medicine 2017;12:231-237. (C) 2017 Society of Hospital Medicine
Hospital-acquired venous thromboembolism (VTE) continues to be a critical quality challenge for U.S. hospitals, ${ }^{1}$ and high-risk patients are often not adequately prophylaxed. Use of VTE prophylaxis (VTEP) varies as widely as $26 \%$ to $85 \%$ of patients in various studies, as does patient outcomes and care expenditures. ${ }^{2-6}$ The 9th edition of the American College of Chest Physicians (CHEST) guidelines ${ }^{7}$ recommend the Padua Prediction Score (PPS) to select individual patients who may be at high risk for venous thromboembolism (VTE) and could benefit from thromboprophylaxis. Use of the manually calculated PPS to select patients for thromboprophylaxis has been shown to help decrease 30-day and 90-day mortality associated with VTE events after hospitalization to medical services. ${ }^{8}$ However, the PPS requires

\footnotetext{
*Address for correspondence and reprint requests: Pierre Elias, MD, Columbia University-New York Presbyterian Hospital, 622 West 168th Street, VC-205, New York, NY 10032; Telephone: 212-305-6354; Fax: 212-305-6279; E-mail: pae9043@nyp.org.

Additional Supporting Information may be found in the online version of this article.

Received: March 13, 2016; Revised: September 26, 2016; Accepted: September 27, 2016
}

2017 Society of Hospital Medicine DOI 10.12788/jhm.2714 time-consuming manual calculation by a provider, who may be focused on more immediate aspects of patient care and several other risk scores competing for his attention, potentially decreasing its use.

Other risk scores that use only discrete scalar data, such as vital signs and lab results to predict early recognition of sepsis, have been successfully automated and implemented within electronic health records (EHRs). ${ }^{9-11}$ Successful automation of scores requiring input of diagnoses, recent medical events, and current clinical status such as the PPS remains difficult. ${ }^{12}$ Data representing these characteristics are more prone to error, and harder to translate clearly into a single data field than discrete elements like heart rate, potentially impacting validity of the calculated result. ${ }^{13}$ To improve usage of guideline based VTE risk assessment and decrease physician burden, we developed an algorithm called Automated Padua Prediction Score (APPS) that automatically calculates the PPS using only EHR data available within prior encounters and the first 4 hours of admission, a similar timeframe to when admitting providers would be entering orders. Our goal was to assess if an automatically calculated version of the PPS, a score that depends on criteria more complex than vital signs and labs, would accurately assess risk for hospital-acquired VTE when compared to 
traditional manual calculation of the Padua Prediction Score by a provider.

\section{METHODS}

\section{Site Description and Ethics}

The study was conducted at University of California, San Francisco Medical Center, a 790-bed academic hospital; its Institutional Review Board approved the study and collection of data via chart review. Handling of patient information complied with the Health Insurance Portability and Accountability Act of 1996.

\section{Patient Inclusion}

Adult patients admitted to a medical or surgical service between July 1, 2012 and April 1, 2014 were included in the study if they were candidates for VTEP, defined as: length of stay (LOS) greater than 2 days, not on hospice care, not pregnant at admission, no present on admission VTE diagnosis, no known contraindications to prophylaxis (eg, gastrointestinal bleed), and were not receiving therapeutic doses of warfarin, low molecular weight heparins, heparin, or novel anticoagulants prior to admission.

\section{Data Sources}

Clinical variables were extracted from the EHR's enterprise data warehouse (EDW) by SQL Server query (Microsoft, Redmond, Washington) and deposited in a secure database. Chart review was conducted by a trained researcher (Mr. Jacolbia) using the EHR and a standardized protocol. Findings were recorded using REDCap (REDCap Consortium, Vanderbilt University, Nashville, Tennessee). The specific ICD-9, procedure, and lab codes used to determine each criterion of APPS are available in the Appendix.

\section{Creation of the Automated Padua Prediction Score (APPS)}

We developed APPS from the original 11 criteria that comprise the Padua Prediction Score: active cancer, previous VTE (excluding superficial vein thrombosis), reduced mobility, known thrombophilic condition, recent (1 month or less) trauma and/or surgery, age 70 years or older, heart and/or respiratory failure, acute myocardial infarction and/ or ischemic stroke, acute infection and/or rheumatologic disorder, body mass index (BMI) 30 or higher, and ongoing hormonal treatment. ${ }^{13}$ APPS has the same scoring methodology as PPS: criteria are weighted from 1 to 3 points and summed with a maximum score of 20 , representing highest risk of VTE. To automate the score calculation from data routinely available in the EHR, APPS checks pre-selected structured data fields for specific values within laboratory results, orders, nursing flowsheets and claims. Claims data included all ICD-9 and procedure codes used for billing purposes. If any of the predetermined data elements are found, then the specific criterion is considered positive; otherwise, it is scored as negative. The creators of the PPS were consulted in the generation of these data queries to replicate the original standards for deeming a criterion pos- itive. The automated calculation required no use of natural language processing.

\section{Characterization of Study Population}

We recorded patient demographics (age, race, gender, BMI), LOS, and rate of hospital-acquired VTE. These patients were separated into 2 cohorts determined by the VTE prophylaxis they received. The risk profile of patients who received pharmacologic prophylaxis was hypothesized to be inherently different from those who had not. To evaluate APPS within this heterogeneous cohort, patients were divided into 2 major categories: pharmacologic vs. no pharmacologic prophylaxis. If they had a completed order or medication administration record on the institution's approved formulary for pharmacologic VTEP, they were considered to have received pharmacologic prophylaxis. If they had only a completed order for usage of mechanical prophylaxis (sequential compression devices) or no evidence of any form of VTEP, they were considered to have received no pharmacologic prophylaxis. Patients with evidence of both pharmacologic and mechanical were placed in the pharmacologic prophylaxis group. To ensure that automated designation of prophylaxis group was accurate, we reviewed 40 randomly chosen charts because prior researchers were able to achieve sensitivity and specificity greater than $90 \%$ with that sample size. ${ }^{14}$

The primary outcome of hospital-acquired VTE was defined as an ICD-9 code for VTE (specific codes are found in the Appendix) paired with a "present on admission = no" flag on that encounter's hospital billing data, abstracted from the EDW. A previous study at this institution used the same methodology and found 212/226 (94\%) of patients with a VTE ICD-9 code on claim had evidence of a hospital-acquired VTE event upon chart review. ${ }^{14}$ Chart review was also completed to ensure that the primary outcome of newly discovered hospital-acquired VTE was differentiated from chronic VTE or history of VTE. Theoretically, ICD-9 codes and other data elements treat chronic VTE, history of VTE, and hospital-acquired VTE as distinct diagnoses, but it was unclear if this was true in our dataset. For 75 randomly selected cases of presumed hospital-acquired VTE, charts were reviewed for evidence that confirmed newly found VTE during that encounter.

\section{Validation of APPS through Comparison to Manual Calculation of the Original PPS}

To compare our automated calculation to standard clinical practice, we manually calculated the PPS through chart review within the first 2 days of admission on 300 random patients, a subsample of the entire study cohort. The largest study we could find had manually calculated the PPS of 1,080 hospitalized patients with a mean PPS of 4.86 (standard deviation [SD], 2.26). ${ }^{15}$ One researcher (Mr. Jacolbia) accessed the EHR with all patient information available to physicians, including admission notes, orders, labs, flowsheets, past medical history, and all prior encounters to calculate and record the PPS. To limit potential score bias, 2 authors (Drs. Elias and Davies) assessed 30 randomly selected charts from the cohort of 300 . 
The standardized chart review protocol mimicked a physician's approach to determine if a patient met a criterion, such as concluding if he/she had active cancer by examining medication lists for chemotherapy, procedure notes for radiation, and recent diagnoses on problem lists. After the original PPS was manually calculated, APPS was automatically calculated for the same 300 patients. We intended to characterize similarities and differences between APPS and manual calculation prior to investigating APPS' predictive capacity for the entire study population, because it would not be feasible to manually calculate the PPS for all 30,726 patients.

\section{Statistical Analysis}

For the 75 randomly selected cases of presumed hospital-acquired VTE, the number of cases was chosen by powering our analysis to find a difference in proportion of $20 \%$ with $90 \%$ power, $\alpha=0.05$ (two-sided). We conducted $\chi^{2}$ tests on the entire study cohort to determine if there were significant differences in demographics, LOS, and incidence of hospital-acquired VTE by prophylaxis received. For both the pharmacologic and the no pharmacologic prophylaxis groups, we conducted 2 -sample Student t tests to determine significant differences in demographics and LOS between patients who experienced a hospital-acquired VTE and those who did not.

For the comparison of our automated calculation to standard clinical practice, we manually calculated the PPS through chart review within the first 2 days of admission on a subsample of 300 random patients. We powered our analysis to detect a difference in mean PPS from 4.86 to 4.36 , enough to alter the point value, with $90 \%$ power and $\alpha=$ 0.05 (two-sided) and found 300 patients to be comfortably above the required sample size. We compared APPS and manual calculation in the 300-patient cohort using: 2-sample Student $t$ tests to compare mean scores, $\chi^{2}$ tests to compare the frequency with which criteria were positive, and receiver operating characteristic (ROC) curves to determine capacity to predict a hospital-acquired VTE event. Pearson's correlation was also completed to assess score agreement between APPS and manual calculation on a per-patient basis. After comparing automated calculation of APPS to manual chart review on the same 300 patients, we used APPS to calculate scores for the entire study cohort $(\mathrm{n}=30,726)$. We calculated the mean of APPS by prophylaxis group and whether hospital-acquired VTE had occurred. We analyzed APPS' ROC curve statistics by prophylaxis group to determine its overall predictive capacity in our study population. Lastly, we computed the time required to calculate APPS per patient. Statistical analyses were conducted using SPSS Statistics (IBM, Armonk, New York) and Python 2.7 (Python Software Foundation, Beaverton, Oregon); 95\% confidence intervals $(\mathrm{CI})$ and $(\mathrm{SD})$ were reported when appropriate.

\section{RESULTS}

Among the 30,726 unique patients in our entire cohort (all patients admitted during the time period who met the study
TABLE 1. Distribution of Patient Characteristics in Cohort

$\begin{array}{ccc}\text { No Pharmacologic } & \text { Pharmacologic } & \\ \text { Prophylaxis } & \text { Prophylaxis } & P \text { value } \\ (n=24,152) & (n=6574) & \end{array}$

\begin{tabular}{lccc} 
Race or Ethnicity (\%) & & & 0.001 \\
White & $13,765(57.0)$ & $3652(55.6)$ & \\
Hispanic or Latino & $3766(15.6)$ & $908(13.8)$ & \\
Asian & $3072(12.7)$ & $993(15.1)$ & \\
Black or African American & $1685(7.0)$ & $745(11.3)$ & \\
Other/declined & $1447(6.0)$ & $140(2.1)$ & \\
Pacific Islander & $319(1.3)$ & $103(1.6)$ & $<0.001$ \\
American Indian & $98(0.4)$ & $33(0.5)$ & $<0.001$ \\
\hline Male (\%) & $10,236(42.4)$ & $3327(50.6)$ & $<0.001$ \\
\hline Age (SD) & $53.2(17.7)$ & $62.7(17.1)$ & $<0.001$ \\
\hline BMl (SD) & $27.7(6.7)$ & $7.1(10.1)$ & $27.3(7.4)$ \\
\hline LOS, d (SD) & $9.8(11.8)$ & $113(0.5)$ & \\
\hline Hospital-acquired VTE (\%) & & & \\
\hline
\end{tabular}

NOTE: Abbreviations: BMI, body mass index; LOS, length of stay; SD, standard deviation; VTE, venous thromboembolism.

criteria), we found 6574 (21.4\%) on pharmacologic (with or without mechanical) prophylaxis, $13,511(44.0 \%)$ on mechanical only, and 10,641 (34.6\%) on no prophylaxis. $\chi^{2}$ tests found no significant differences in demographics, LOS, or incidence of hospital-acquired VTE between the patients who received mechanical prophylaxis only and those who received no prophylaxis (Table 1). Similarly, there were no differences in these characteristics in patients receiving pharmacologic prophylaxis with or without the addition of mechanical prophylaxis. Designation of prophylaxis group by manual chart review vs. our automated process was found to agree in categorization for 39/40 (97.5\%) sampled encounters. When comparing the cohort that received pharmacologic prophylaxis against the cohort that did not, there were significant differences in racial distribution, sex, BMI, and average LOS as shown in Table 1. Those who received pharmacologic prophylaxis were found to be significantly older than those who did not (62.7 years versus 53.2 years, $P<0.001)$, more likely to be male $(50.6 \%$ vs, $42.4 \%, P<$ $0.001)$, more likely to have hospital-acquired VTE ( $2.2 \%$ vs. $0.5 \%, P<0.001$ ), and to have a shorter LOS ( 7.1 days vs. $9.8, P<0.001)$.

Within the cohort group receiving pharmacologic prophylaxis $(n=6574)$, hospital-acquired VTE occurred in patients who were significantly younger ( 58.2 years vs. 62.8 
TABLE 2. Comparison of APPS to Manual Calculation of PPS

\begin{tabular}{lccc}
\hline & $\begin{array}{c}\text { Manual } \\
\text { Calculation } \\
(\mathrm{n}=300)\end{array}$ & $\begin{array}{c}\text { APPS } \\
(\mathrm{n}=300)\end{array}$ & $P$ \\
\hline Score criteria (\%) & $96(32.0)$ & $39(13.0)$ & $<0.001$ \\
Active cancer $^{3}$ & $25(8.3)$ & $48(16.0)$ & $<0.001$ \\
Prior VTE $^{3}$ & $198(66.0)$ & $223(74.3)$ & $<0.001$ \\
Reduced mobility $^{3}$ & 0 & $17(5.7)$ & $<0.001$ \\
Thrombophilia $^{3}$ & $163(54.3)$ & $117(39.0)$ & $<0.001$ \\
Recent trauma or surgery $^{2}$ & $76(25.3)$ & $77(25.7)$ & 0.655 \\
Age $^{2} 70$ y $^{1}$ & $27(9.0)$ & $66(22.0)$ & $<0.001$ \\
Heart or respiratory failure $^{1}$ & $16(5.3)$ & $54(18.0)$ & $<0.001$ \\
Acute Ml/stroke $^{1}$ & $75(25.0)$ & $57(19.0)$ & 0.036 \\
Acute infection/rheumatic flare $^{1}$ & $42(14.0)$ & $124(41.3)$ & $<0.001$ \\
Obese $^{1}$ & $1(0.3)$ & $5(1.7)$ & 0.103 \\
Ongoing hormonal treatment $^{1}$ & $5.1(2.6)$ & $5.5(2.9)$ & 0.073 \\
\hline Total score (SD) $^{2}$ & & & \\
\hline Hospital-acquired VTE (\%) $^{2}$ & & & \\
\hline
\end{tabular}

${ }^{3,2,1}$ Corresponds to the point value of each criterion in the original PPS and APPS.

NOTE: Abbreviations: APPS, Automated Padua Prediction Score; MI, myocardial infarction; PPS, Padua Prediction Score; SD, standard deviation; VTE, venous thromboembolism. years, $P=0.003)$ with a greater $\operatorname{LOS}(23.8$ days vs. $6.7, P$ $<0.001)$ than those without. Within the group receiving no pharmacologic prophylaxis $(\mathrm{n}=24,152)$, hospital-acquired VTE occurred in patients who were significantly older (57.1 years vs. 53.2 years, $P=0.014$ ) with more than twice the LOS (20.2 days vs. 9.7 days, $P<0.001)$ compared to those without. Sixty-six of $75(88 \%)$ randomly selected patients in which new VTE was identified by the automated electronic query had this diagnosis confirmed during manual chart review.

As shown in Table 2, automated calculation on a subsample of 300 randomly selected patients using APPS had a mean of $5.5(\mathrm{SD}, 2.9)$ while manual calculation of the original PPS on the same patients had a mean of 5.1 (SD, 2.6). There was no significant difference in mean between manual calculation and APPS $(P=0.073)$. There were, however, significant differences in how often individual criteria were considered present. The largest contributors to the difference in scores between APPS and manual calculation were "prior VTE" (positive, $16 \%$ vs. $8.3 \%$, respectively) and "reduced mobility" (positive, $74.3 \%$ vs. $66 \%$, respectively) as shown in Table 2 . In the subsample, there were a total of $6(2.0 \%)$ hospital-acquired VTE events. APPS' automated calculation had an AUC $=0.79(\mathrm{CI}, 0.63-0.95)$ that was significant $(P=0.016)$ with a cutoff value of 5 . Chart review's manual calculation of the PPS had an AUC $=0.76$ (CI 0.61$0.91)$ that was also significant $(P=0.029)$.

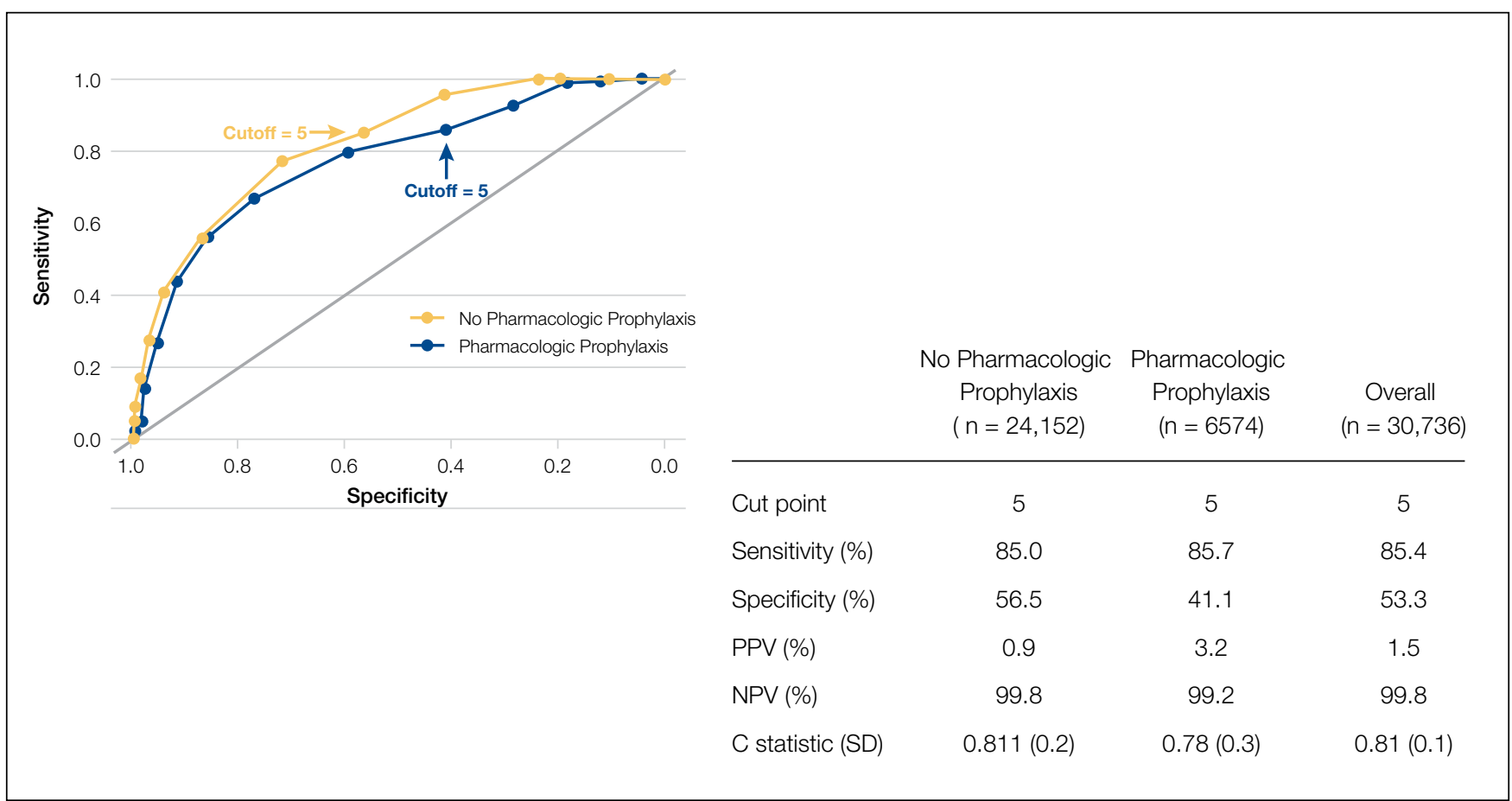

FIG. (Left) ROC curves of the APPS predicting hospital-acquired VTE of patients receiving no pharmacologic prophylaxis (yellow line, $n=24,152$ ) vs pharmacologic prophylaxis (blue line, $n=6574$ ). (Right) Predictive characteristics of APPS based on prophylaxis received and for entire cohort. Each AUC was significantly greater than reference of $0.5(P<0.001)$.

NOTE: Abbreviations: APPS, Automated Padua Prediction Score; AUC, area under the curve; NPV, negative predictive value; PPV, positive predictive value; ROC, receiver operating characteristic; SD, standard deviation; VTE, venous thromboembolism 
Our entire cohort of 30,726 unique patients admitted during the study period included $260(0.8 \%)$ who experienced hospital-acquired VTEs (Table 3). In patients receiving no pharmacologic prophylaxis, the average APPS was 4.0 (SD, 2.4) for those without VTE and 7.1 (SD, 2.3) for those with VTE. In patients who had received pharmacologic prophylaxis, those without hospital-acquired VTE had an average APPS of 4.9 (SD, 2.6) and those with hospital-acquired VTE averaged 7.7 (SD, 2.6). APPS' ROC curves for "no pharmacologic prophylaxis" had an $\mathrm{AUC}=0.81(\mathrm{CI}, 0.79-0.83)$ that was significant $(P<0.001)$ with a cutoff value of 5 . There was similar performance in the pharmacologic prophylaxis group with an $\mathrm{AUC}=0.79$ (CI, $0.76-$ 0.82 ) and cutoff value of 5 , as shown in the Figure. Over the entire cohort, APPS had a sensitivity of $85.4 \%$, specificity of $53.3 \%$, positive predictive value (PPV) of $1.5 \%$, and a negative predictive value (NPV) of $99.8 \%$ when using a cutoff of 5 . The average APPS calculation time was 0.03 seconds per encounter. Additional information on individual criteria can be found in Table 3.

\section{DISCUSSION}

Automated calculation of APPS using EHR data from prior encounters and the first 4 hours of admission was predictive of in-hospital VTE. APPS performed as well as traditional manual score calculation of the PPS. It was able to do so with no physician input, significantly lessening the burden of calculation and potentially increasing frequency of data-driven VTE risk assessment.

While automated calculation of certain scores is becoming more common, risk calculators that require data beyond vital signs and lab results have lagged, ${ }^{16-19}$ in part because of uncertainty about 2 issues. The first is whether EHR data accurately represent the current clinical picture. The second is if a machine-interpretable algorithm to determine a clinical status (eg, "active cancer") would be similar to a doctor's perception of that same concept. We attempted to better understand these 2 challenges through developing APPS. Concerning accuracy, EHR data correctly represent the clinical scenario: designations of VTEP and hospital-acquired VTE were accurate in approximately $90 \%$ of reviewed cases. Regarding the second concern, when comparing APPS to manual calculation, we found significant differences $(P<$ 0.001 ) in how often 8 of the 11 criteria were positive, yet no significant difference in overall score and similar predic- tive capacity. Manual calculation appeared more likely to find data in the index encounter or in structured data. For example, "active cancer" may be documented only in a physician's note, easily accounted for during a physician's calculation but missed by APPS looking only for structured data. In contrast, automated calculation found historic criteria, such as "prior VTE" or "known thrombophilic condition," positive more often. If the patient is being admitted for a problem unrelated to blood clots, the physician may have little time or interest to look through hundreds of EHR documents to discover a 2-year-old VTE. As patients' records become larger and denser, more historic data can become buried and forgotten. While the 2 scores differ on individual criteria, they are similarly predictive and able to bifurcate the at-risk population to those who should and should not receive pharmacologic prophylaxis.

The APPS was found to have near-equal performance in the pharmacologic vs. no pharmacologic prophylaxis cohorts. This finding agrees with a study that found no sig- 
nificant difference in predicting 90-day VTE when looking at 86 risk factors vs. the most significant 4 , none of which related to prescribed prophylaxis. ${ }^{18}$ The original PPS had a reported sensitivity of $94.6 \%$, specificity $62 \%$, PPV $7.5 \%$, and NPV $99.7 \%$ in its derivation cohort. ${ }^{13}$ We matched APPS to the ratio of sensitivity to specificity, using 5 as the cutoff value. APPS performed slightly worse with sensitivity of $85.4 \%$, specificity $53.3 \%$, PPV $1.5 \%$, and NPV $99.8 \%$. This difference may have resulted from the original PPS study's use of 90-day follow-up to determine VTE occurrence, whereas we looked only until the end of current hospitalization, an average of 9.2 days. Furthermore, the PPS had significantly poorer performance $($ AUC $=0.62$ ) than that seen in the original derivation cohort in a separate study that manually calculated the score on more than 1000 patients. ${ }^{15}$

There are important limitations to our study. It was done at a single academic institution using a dataset of VTE-associated, validated research that was well-known to the researchers. ${ }^{20}$ Another major limitation is the dependence of the algorithm on data available within the first 4 hours of admission and earlier; thus, previous encounters may frequently play an important role. Patients presenting to our health system for the first time would have significantly fewer data available at the time of calculation. Additionally, our data could not reliably tell us the total doses of pharmacologic prophylaxis that a patient received. While most patients will maintain a consistent VTEP regimen once initiated in the hospital, 2 patients with the same LOS may have received differing amounts of pharmacologic prophylaxis. This research study did not assess how much time automatic calculation of VTE risk might save providers, because we did not record the time for each manual abstraction; however, from discussion with the main abstracter, chart review and manual calculation for this study took from 2 to 14 minutes per patient, depending on the number of previous interactions with the health system. Finally, although we chose data elements that are likely to exist at most institutions using an EHR, many institutions' EHRs do not have EDW capabilities nor programmers who can assist with an automated risk score.

The EHR interventions to assist providers in determining appropriate VTEP have been able to increase rates of VTEP and decrease VTE-associated mortality. ${ }^{16,21}$ In addition to automating the calculation of guideline-adherent risk scores, there is a need for wider adoption for clinical decision support for VTE. For this reason, we chose only structured data fields from some of the most common elements within our EHR's data warehouse to derive APPS (Appendix 1). Our study supports the idea that automated calculation of scores requiring input of more complex data such as diagnoses, recent medical events, and current clinical status remains predictive of hospital-acquired VTE risk. Because it is calculated automatically in the background while the clinician completes his or her assessment, the APPS holds the potential to significantly reduce the burden on providers while making guideline-adherent risk assessment more readily accessible. Further research is required to determine the exact amount of time automatic calculation saves, and, more important, if the relatively high predictive capacity we observed using APPS would be reproducible across institutions and could reduce incidence of hospitalacquired VTE.

Disclosures: Dr. Auerbach was supported by NHLBI K24HL098372 during the period of this study. Dr. Khanna, who is an implementation scientist at the University of California San Francisco Center for Digital Health Innovation, is the principal inventor of CareWeb, and may benefit financially from its commercialization. The other authors report no financial conflicts of interest.

\section{References}

1. Galson S. The Surgeon General's call to action to prevent deep vein thrombosis and pulmonary embolism. 2008. http://www.surgeongeneral.gov/library/calls/ deepvein/call-to-action-on-dvt-2008.pdf. Accessed February 11, 2016.

2. Borch KH, Nyegaard C, Hansen JB, et al. Joint effects of obesity and body height on the risk of venous thromboembolism: the Troms $\varnothing$ study. Arterioscler Thromb Vasc Biol. 2011;31(6):1439-44.

3. Braekkan SK, Borch KH, Mathiesen EB, Njølstad I, Wilsgaard T, Hansen JB.. Body height and risk of venous thromboembolism: the Troms $\varnothing$ Study. Am J Epidemiol. 2010;171(10):1109-1115.

4. Bounameaux H, Rosendaal FR. Venous thromboembolism: why does ethnicity matter? Circulation. 2011;123(200:2189-2191.

5. Spyropoulos AC, Anderson FA Jr, Fitzgerald G, et al; IMPROVE Investigators. Predictive and associative models to identify hospitalized medical patients at risk for VTE. Chest. 2011;140(3):706-714.

6. Rothberg MB, Lindenauer PK, Lahti M, Pekow PS, Selker HP. Risk factor model to predict venous thromboembolism in hospitalized medical patients. J Hosp Med. 2011;6(4):202-209.

7. Perioperative Management of Antithrombotic Therapy: Prevention of VTE in Nonsurgical Patients: Antithrombotic Therapy and Prevention of Thrombosis, 9th ed: American College of Chest Physicians Evidence-Based Clinical Practice Guidelines. Chest. 2012;141(6):1645.

8. Subbe CP, Kruger M, Rutherford P, Gemmel L. Validation of a modified Early Warning Score in medical admissions. QJM. 2001;94(10):521-526.

9. Alvarez CA, Clark CA, Zhang S, et al. Predicting out of intensive care unit cardiopulmonary arrest or death using electronic medical record data. BMC Med Inform Decis Mak. 2013;13:28.

10. Escobar GJ, LaGuardia JC, Turk BJ, Ragins A, Kipnis P, Draper D. Early detection of impending physiologic deterioration among patients who are not in intensive care: development of predictive models using data from an automated electronic medical record. J Hosp Med. 2012;7(5):388-395.

11. Umscheid CA, Hanish A, Chittams J, Weiner MG, Hecht TE. Effectiveness of a novel and scalable clinical decision support intervention to improve venous thromboembolism prophylaxis: a quasi-experimental study. BMC Med Inform Decis Mak. 2012;12:92.

12. Tepas JJ 3 ${ }^{\text {rd }}$, Rimar JM, Hsiao AL, Nussbaum MS. Automated analysis of electronic medical record data reflects the pathophysiology of operative complications. Surgery. 2013;154(4):918-924.

13. Barbar S, Noventa F, Rossetto V, et al. A risk assessment model for the identification of hospitalized medical patients at risk for venous thromboembolism: the Padua Prediction Score. J Thromb Haemost. 2010; 8(11):2450-2457.

14. Khanna R, Maynard G, Sadeghi B, et al. Incidence of hospital-acquired venous thromboembolic codes in medical patients hospitalized in academic medical centers. J Hosp Med. 2014; 9(4):221-225.

15. Vardi M, Ghanem-Zoubi NO, Zidan R, Yurin V, Bitterman H. Venous thromboembolism and the utility of the Padua Prediction Score in patients with sepsis admit- 
ted to internal medicine departments. J Thromb Haemost. 2013;11(3):467-473.

16. Samama MM, Dahl OE, Mismetti P, et al. An electronic tool for venous thromboembolism prevention in medical and surgical patients. Haematologica. 2006;91(1):64-70

17. Mann DM, Kannry JL, Edonyabo D, et al. Rationale, design, and implementation protocol of an electronic health record integrated clinical prediction rule (iCPR) randomized trial in primary care. Implement Sci. 2011;6:109.

18. Woller SC, Stevens SM, Jones JP, et al. Derivation and validation of a simple model to identify venous thromboembolism risk in medical patients. Am J Med.
2011;124(10):947-954.

19. Huang W, Anderson FA, Spencer FA, Gallus A, Goldberg RJ. Risk-assessment models for predicting venous thromboembolism among hospitalized non-surgical patients: a systematic review. J Thromb Thrombolysis. 2013;35(1):67-80.

20. Khanna RR, Kim SB, Jenkins I, et al. Predictive value of the present-on-admission indicator for hospital-acquired venous thromboembolism. Med Care. 2015;53(4):e31-e36.

21. Kucher N, Koo S, Quiroz R, et al. Electronic alerts to prevent venous thromboembolism among hospitalized patients. N Engl J Med. 2005;352(10):969-977. 